\title{
Concept Design and Reliability
}

G. Cooper, G. Thompson

The paper outlines an approach to concept design that integrates the development of the design specification with multi-objective concept development. 'Excursions' are undertaken to explore particular aspects of the design, e.g., reliability, in order to help create a concept that is strong in the salient design requirements.

The paper then considers reliability as a principal design requirement. Existing reliability evaluation methods are reviewed with respect to their suitability for use in concept design. The paper then describes an approach, an 'excursion', that can be used to improve the reliability of a concept. The reliability excursion comprises a systematic method of evaluation and a reliability analysis toolbox. The research reported is an element of a larger concept design activity that itself forms part of a substantial project on integrated product design undertaken at the Polhem Laboratory, Lule Technical University, Sweden and at UMIST, UK.

Keywords: conceptual design, reliability, approximate analysis tools.

\section{Introduction}

All stages of design within the design process are important, however it can be argued that the early stages of design contribute more significantly to the success or failure of the fi-

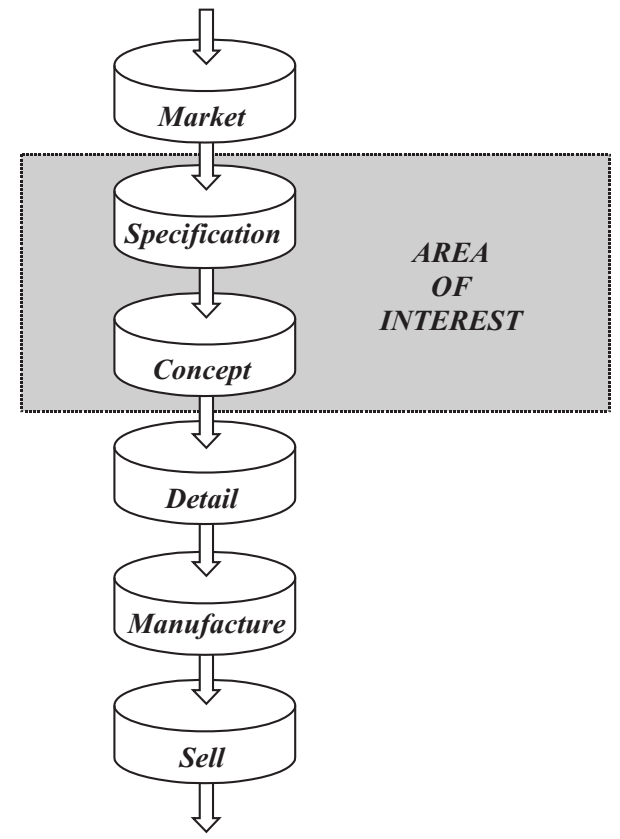

Fig. 1: Simplification of Pugh's total design model, showing area of interest nal product or system. If a specification or concept is poorly defined or conceived then it is virtually impossible to improve the design when it is taken to the detail stage. In order to improve the functionality, reliability and maintainability of designs it is suggested that research be directed at the earlier stages of design. It is the decisions taken during design that greatly affect the cost of products and the early, conceptual stage of design has a major influence on the outcome of design activities. Pugh's total design model illustrates the design process as it is conventionally understood. However, for the purposes of this research only the specification and conceptual design stage will be studied, the early stages. This is shown in Fig. 1 [1].

Fig. 2 illustrates a summary of the design process that is used as the basis this research. It is an integrated product design process that is presently being pursued as a collaborative activity between the Polhem Laboratory, Lule Technical University, Sweden and UMIST, UK in collaboration with major manufacturing industries in Sweden.

Derivation of the specification and concept design is an integrated activity. Working from a 'seed specification', an immature concept is derived that is evaluated using appropriate methods. From the evaluation, the specification and the concept are developed further and then analysed. Thus concept design becomes a set of divergent-convergent design activities that develop an immature concept to maturity by strengthening the concept in response to the salient design requirements.

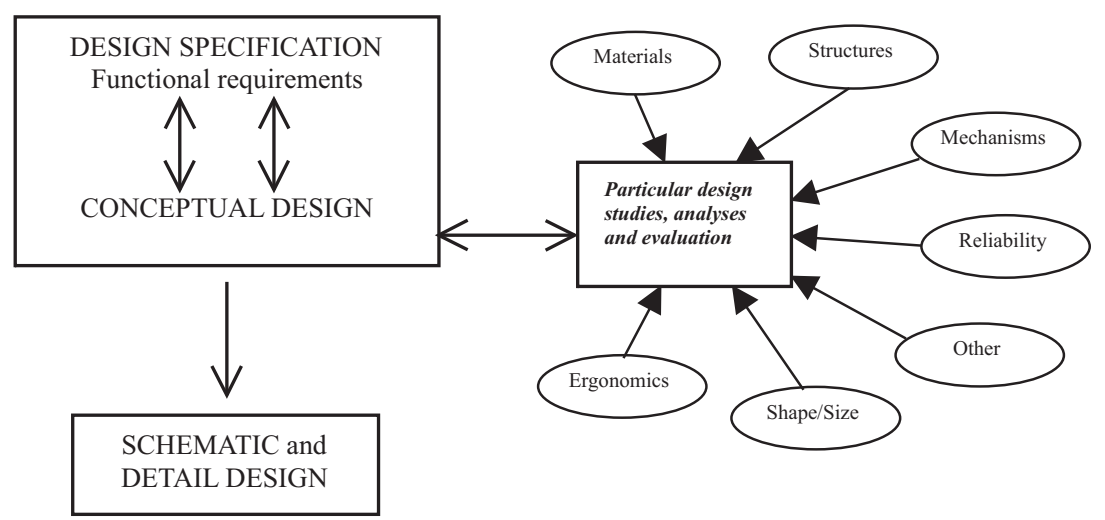

Fig. 2: Summary of the early design process 
The seed specification is intended to give guidance to the customer, when producing the product design specification, PDS, and to the design team, when developing the initial conceptual designs. It is intended that the seed specification is fixed early in the design process. The full PDS can then grow with the conceptual design phase. Iteration and changes to the specification and conceptual design is expected and encouraged during this early design phase. It is within these early design phases that changes and iterations can and should be made. There is little expense involved in investigating alternative solutions and strategies when the design is still in the idea stage (that is, no prototypes or detail design has been undertaken). The process of this early design phase is demonstrated by figure 1.3. Note that the seed specification is at the heart of the process and it is from this that the design grows. Also the development of the specification and concept flow into each other and grow together. Each process feeds and iterates from each other until the full PDS and conceptual solution emerges. Only now should the process provide an output to the next stage. If the concept and specification are not developed fully and effectively before moving to detail design it is almost inevitable that later design problems will occur.

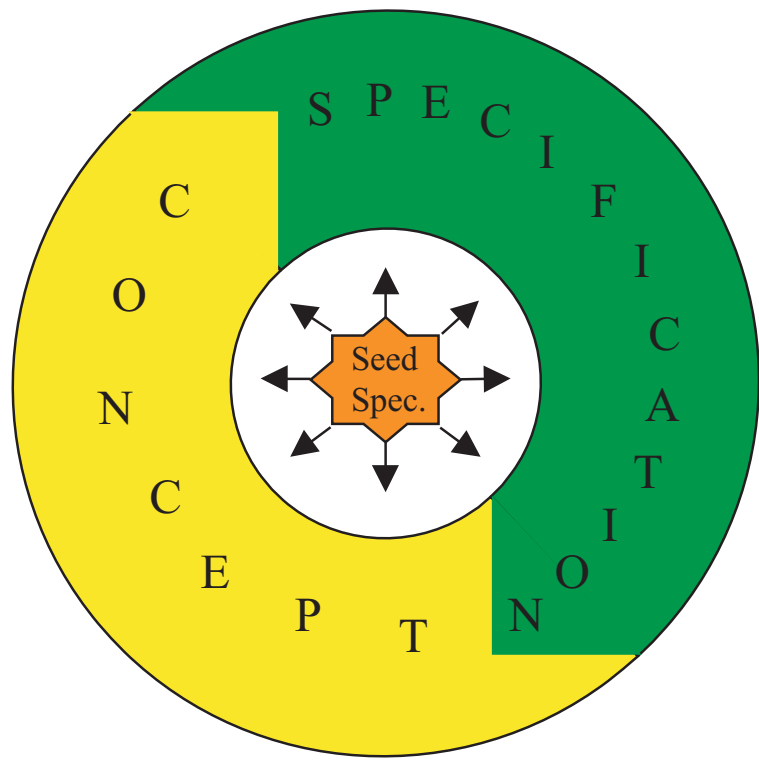

Fig. 3: The early design process

As part of the concept design activity, excursions are made to investigate particular aspects of the design, e.g. a particular material performance requirement, feasibility of manufacture, reliability. The objective of the excursion is not to undertake detail design but rather to investigate feasibility or to make improvements in order to create a concept with strong attributes. Once the concept is considered feasible, then no iteration is envisaged back from the schematic or detail design phase. This is considered important since the intention is to make the design 'right first time' and to minimise the time to production.

In order to develop the design concept effectively a number of tools, both divergent and convergent are required for use in the excursions. Since the tools are to be used at the conceptual stage, there may be little detailed information available.
Excursions are currently made to select suitable materials for certain performance criteria. It may be that the material must be compatible with others in its surroundings, or it might have to endure harsh environmental conditions such as high temperature, corrosive chemicals or high voltages. Also a variety of loading conditions may dictate material choice. Excursions are currently available to prove feasibility and make judgements regarding material choice. Similarly, simple structural calculations can be performed to prove that the required loading conditions can be met. If mechanisms are part of the design simple analysis can be undertaken to ensure that they will operate correctly. Where human interaction is required with a product, ergonomic considerations can be made effectively by using the relevant judgement and past data if so required.

All the aforementioned excursions can be performed effectively with the current tools and techniques available to designers. However, if reliability is to be investigated at this early design stage it is found that only highly analytical tools exist (in the majority of cases). These analytical tools require detailed information, therefore their use is not immediately accepted as useful in conceptual design. Because of this reliability assessment is often neglected until the later design stages, such as detail design. If this situation could be avoided and use could be made of a reliability excursion in the early design stages then it is hypothesised that product design would be greatly improved.

This paper is concerned with excursions that contribute to the formation of a concept with strong reliability attributes or to improve the reliability of a concept.

\section{Reliability analysis tools}

There are many analytical tools that are used in the design of equipment and products. These tools are used to increase the likelihood that the equipment/product will perform its specified functions, in other words to increase the reliability that the products' functions will be provided. Table 1 lists the reliability tools that have been looked at within the literature review and some additional methods are included. It is appreciated that these are a diverse set of techniques and that they are non-homogeneous. However, this is the nature of reliability tools and techniques. The authors felt the need to consider many different approaches before developing specific methodology. Even the list within Table 1 is not exhaustive but it is intended to be as inclusive as possible [2, 3, $4,5,6,7,8,9,10,11]$.

To create a product that will perform a function reliably the reliability must be considered as an important functional requirement all the way through the design process, from the customer's need to the final product. It can be seen from Table 1 that the majority of the tools available for reliability analysis are for use at the detail/schematic or post design. However, reliability is significantly influenced at other stages of design.

The customer should be made aware and educated that specific reliability requirements should be incorporated and valued equally with other functional requirements in the specification. In particular when contracts are at the tender stage, cost should not be the sole selection factor for the customer or project engineer. In conceptual design, 
functional performance is often considered more important than reliability. Reliability is hard to quantify at the concept stage. Many of the present reliability analysis methods require precise engineering information for their implementation making them inappropriate for use in early concept design. Increased pressure is also placed on the designer to reduce the initial purchase costs of products when the emphasis of cost should preferably lie on the overall cost for the full lifetime of the product. Life-cycle cost considerations in design place a high emphasis on reliability.

Design for maintainability and design for manufacture and assembly will also increase the products' reliability. These factors should be considered and implemented from the specification to detail design stages to produce equipment that will not incur reliability problems during manufacture and servicing. PSolutions must be found to solve the problem

Table 1: Reliability Analysis Tools

\begin{tabular}{|c|c|c|c|c|c|c|}
\hline 1 & Accelerated testing & $\mathrm{D}$ & 27 & Mission profile development & $\mathrm{S}$ & \\
\hline 2 & Availability analysis \& modelling & $\mathrm{D}, \mathrm{C}$ & 28 & MTTF \& MTTR & $\mathrm{P}, \mathrm{D}$ & \\
\hline 3 & Baysian belief nets & $\mathrm{P}$ & 29 & Pareto Analysis & $\mathrm{P}$ & \\
\hline 4 & Binary decision diagrams & $\mathrm{P}$ & 30 & Part derating & C, D & $\mathrm{f}$ \\
\hline 5 & Business process analysis & $\mathrm{B}$ & 31 & $\begin{array}{l}\text { Physics of failure (\& computational } \\
\text { methods) }\end{array}$ & $\mathrm{D}$ & \\
\hline 6 & Cause consequence diagrams & $\mathrm{P}$ & 32 & Probabilistic design & $\mathrm{D}, \mathrm{C}$ & \\
\hline 7 & Classical design of experiments & $\mathrm{D}$ & 33 & Pugh's concept selection process & $\mathrm{C}$ & \\
\hline 8 & Creative problem solving & $\mathrm{P}, \mathrm{D} \quad \mathrm{e}$ & 34 & Quality function deployment & $\mathrm{D}, \mathrm{C}$ & \\
\hline 9 & Damage tolerant design & $\mathrm{D}, \mathrm{C} \quad \mathrm{f}$ & 35 & Reliability allocation & $\mathrm{D}, \mathrm{C}, \mathrm{S}$ & $\mathrm{i}$ \\
\hline 10 & Data base development & $\mathrm{g}$ & 36 & Reliability benchmarking & $\mathrm{D}, \mathrm{C}, \mathrm{S}$ & $\mathrm{i}$ \\
\hline 11 & $\begin{array}{l}\text { Device performance index } \& \text { multi } \\
\text { objective method }\end{array}$ & $\mathrm{P}$ & 37 & Reliability block diagram development & $\mathrm{D}, \mathrm{C}$ & \\
\hline 12 & Environment \& product usage profile & $\mathrm{S}$ & 38 & $\begin{array}{l}\text { Reliability centred and risk based } \\
\text { maintenance }\end{array}$ & $\mathrm{D}$ & \\
\hline 13 & Environment stress screening & $\mathrm{S}$ & 39 & Reliability demonstration testing & $\mathrm{D}$ & $\mathrm{a}$ \\
\hline 14 & $\begin{array}{l}\text { Error/mistake proofing (quality } \\
\text { procedure) }\end{array}$ & $\mathrm{B}$ & 40 & Reliability growth testing & $\mathrm{D}$ & a \\
\hline 15 & Event tree analysis & $\mathrm{P}$ & 41 & Reliability prediction & $\mathrm{P}, \mathrm{D}$ & \\
\hline 16 & Expert design review & $\mathrm{D}, \mathrm{C} \quad \mathrm{h}$ & 42 & Risk analysis & $\mathrm{P}, \mathrm{D}$ & $\mathrm{k}$ \\
\hline 17 & $\begin{array}{l}\text { Failure reporting \& corrective action } \\
\text { system }\end{array}$ & $\mathrm{D}$ & 43 & Robust design & $\mathrm{S}, \mathrm{C}$ & $\mathrm{f}$ \\
\hline 18 & Fault tolerant design & $\mathrm{D}, \mathrm{C} \quad \mathrm{f}$ & 44 & Root cause analysis & $\mathrm{P}, \mathrm{D}$ & $\mathrm{e}$ \\
\hline 19 & FMEA \& FMECA & $\mathrm{D}, \mathrm{C}$ & 45 & Sampling procedure & $\mathrm{D}$ & $\mathrm{g}$ \\
\hline 20 & Focus groups & $\mathrm{D}, \mathrm{C}, \mathrm{S} \quad \mathrm{h}$ & 46 & Surveys \& market analysis & $\mathrm{S}$ & \\
\hline 21 & Fault Tree Analysis & $\mathrm{D}, \mathrm{C}$ & 47 & TRIZ & $\mathrm{P}$ & \\
\hline 22 & Functional failure analysis & $\mathrm{D}, \mathrm{C}$ & 48 & Variation simulation modelling & $\mathrm{P}, \mathrm{D}$ & \\
\hline 23 & Graph theory & $\mathrm{C}$ & 49 & Vulnerability analysis & $\mathrm{P}, \mathrm{D}$ & $\mathrm{k}$ \\
\hline 24 & Hazop & $\mathrm{P}, \mathrm{D}$ & 50 & $\begin{array}{l}\text { Warranty tracking \& information } \\
\text { collection }\end{array}$ & $\mathrm{D}$ & $\mathrm{g}$ \\
\hline 25 & HiP-HOPS & $\mathrm{C}$ & 51 & Weibull analysis & $\mathrm{D}$ & \\
\hline 26 & Lessons learned analysis & $\mathrm{D}$ & 52 & Worst case analysis & $\mathrm{P}, \mathrm{D}$ & \\
\hline
\end{tabular}

$\mathrm{D}$ - used in detail or post design

$\mathrm{P}$ - shows potential for adaptation for use at the conceptual design stage

$\mathrm{C}$ - used at the conceptual design stage

$\mathrm{S}$ - used at the specification stage

$\mathrm{B}$ - business process analysis tool

a,b ..., k-indicate similar techniques 
of producing more reliable products. It is suggested that the lack of appropriate reliability analysis tools that can use approximate information at the conceptual design stage is one of the main causes of the problem.

It is hypothesised that if the tools are broken down with regard to their function then it may be easier to take their good attributes and combine them with other tools that may lead to the development of better analysis techniques and methods. Below Fig. 4 shows how the reliability tools have been broken down into 10 functional groups.

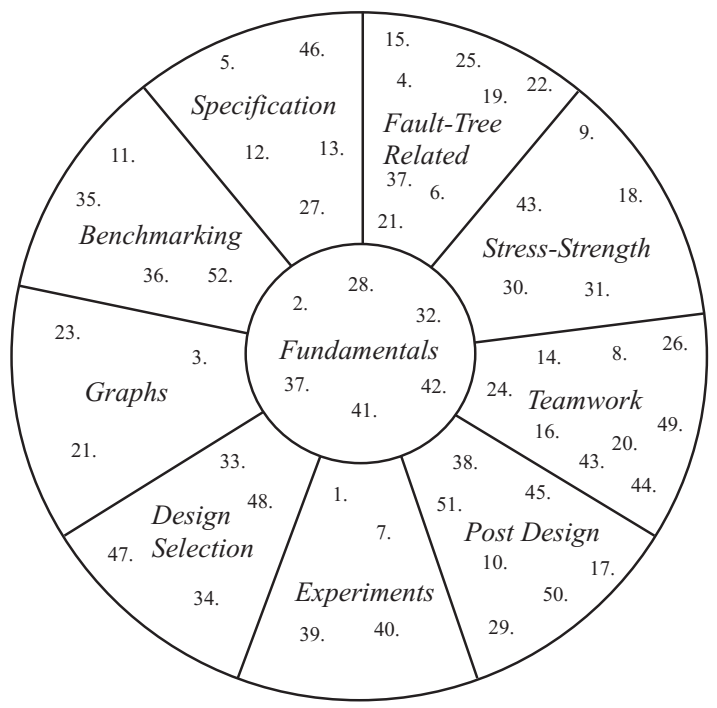

Fig. 4: Grouped tools, for number reference see Table 1

By studying tools within functional groups with good and promising attributes it is hoped that better ways to use existing tools and new tools will emerge.

\section{RA Toolbox for designers}

A system, the Reliability Analysis Toolbox, is proposed to facilitate reliability analyses in concept design. The RA Toolbox will provide a systematic methodology for modelling and improving the reliability of concepts that do not have detailed performance and design data associated with them.

\subsection{Tools}

The main tools considered within this study have been taken from the Table in section 2. Although only a few of these tools have been chosen it is not anticipated that the other tools researched should be ignored. All the tools have advantages and it is envisaged that lessons will be learned from the range of tools. This will then support the development and use of the new techniques studied within this research.

Graphical methods provide a simple way for the designer to consider system elements and their interactions. It for this reason that Graph theory [7] and Bayesian Belief Networks [8] will be studied and used to form the basis of some new tools and techniques. Complex matrix manipulation and probability data can be manipulated and used within these methods. However, for the simple analysis tools to be developed it envisaged that simple data will be handled by the methods to aid approximate feasibility analysis of conceptual design solutions.

A few traditional methods can handle reliability considerations in the early design phases. Fault trees [4] and some related methods are an example of the few. Fault trees and the related techniques form a very powerful way to analyse designs and it is hoped that these can be used to support and develop new techniques. In addition it may be possible to discover new applications of these root reliability analysis tools.

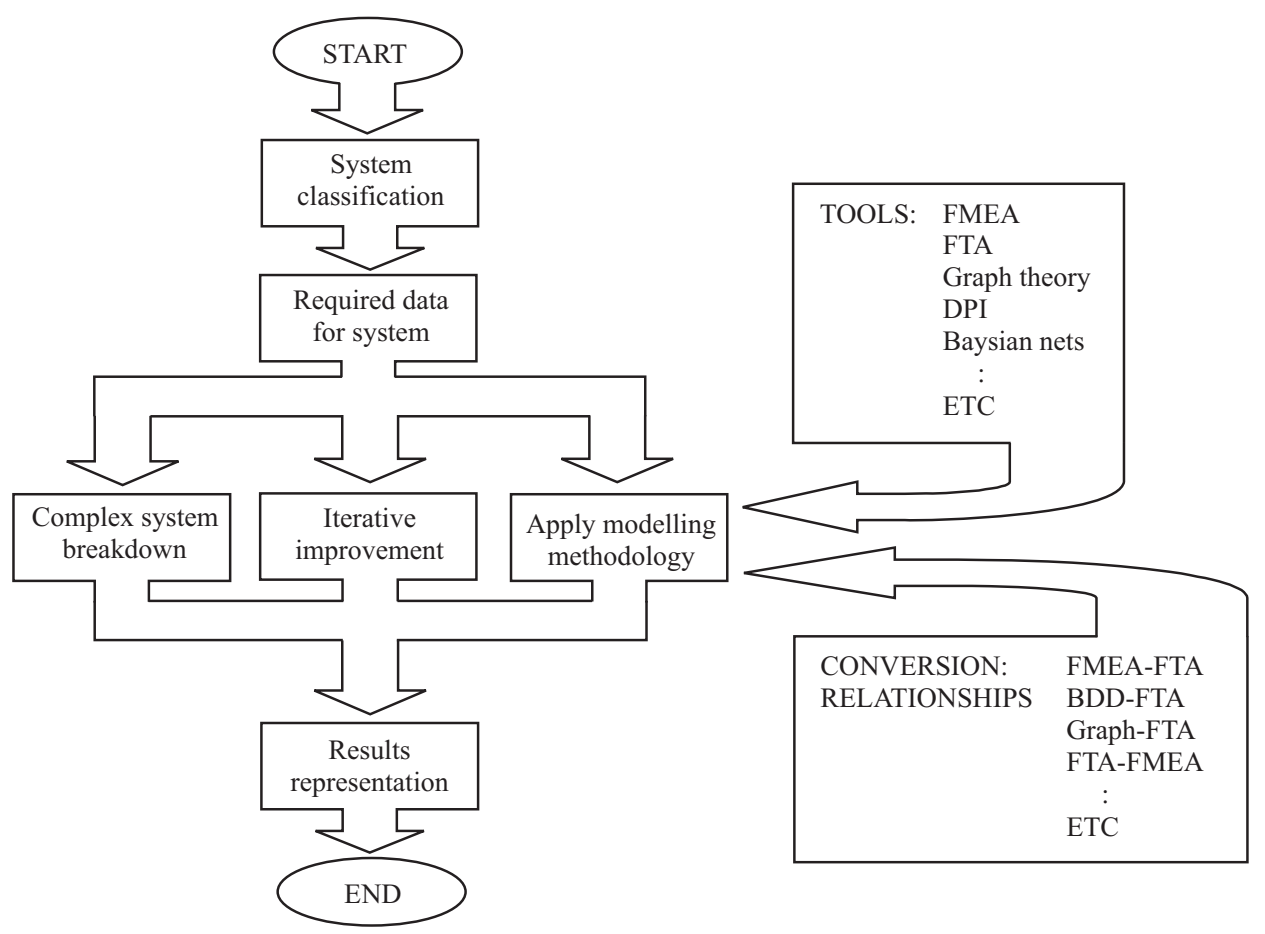

Fig. 5: Reliability Analysis Toolbox 
The multi-objective method [9] is a promising technique which uses approximate data to study the system. It is anticipated that emulating this technique will provide a means to gain input data.

Finally the Robust design ideals of Carter [12] and Taguchi [13] are thought by the author to be crucial in developing a reliable design. It is hoped that these concepts will be extended throughout the tools studied within this project.

Analysis tools are only one aspect of the improvement of reliability. It is equally necessary to consider when and how to implement them, which raises the need for a methodology for conceptual reliability analysis. Fig. 5 shows the basic concept for a design methodology (RA Toolbox) and below, a description is given of the various functions within it.

The methodology will take one of the three possible routes through the model it will not attempt to complete steps in parallel. However, it will be possible to use the methodology more than once to complete all the three routes for a design from its beginning to end.

\subsection{Conversion relationships}

FMEA can be difficult to interpret if the study spans many pages. However, FTA is usually simpler to understand at a glance, hence conversion methodology [14] (sometimes in the form of computer packages) exist to transform from FMEA to FTA and vice versa. When studying FTA it is found that it can be converted to BDD quite easily. It is clear that many of these relationships exist but have not been investigated and exploited fully.

It is hoped that by finding relationships between the methods it will become easier to use them in collaboration with each other. Also it may be useful to utilise the conversion relationships to extract the good points from each tool to combine them into new tools and analysis methodologies. This will then enable the option for one tool to be used for processing of data and another to be used for displaying results if so desired. Also it may become apparent that a lot of methods are connected and can be converted (which is presently hypothesised).

\subsection{Complex system breakdown}

Breaking down the system into subsystems and knowing the overall system reliability requirement could generate reliability requirements for the subsystems. This method could then be iterated through to the component level. Also reference [6] suggests that HiP-HOPS could be used to break down a complex drive by wire system and demonstrates that system complexity need not create problems in modelling system reliability.

In addition to this, it should be considered that systems are often broken down into subsystems when in fact it may be better to break down the requirement (from the specification) into functions to be performed by the system. This would then allow the designer to consider a greater number of options to perform the functions before selecting the subsystem to perform it. This may be especially applicable to large projects that require a number of companies to bid for contract to produce the subsystems. Reliability could then be assessed by the ability to perform that specified function and in turn used to with other criteria to select the best concept to provide the overall function.

\subsection{System classification}

It is impossible to develop an analysis methodology that will be fully comprehensive to all types of concept. Instead, methodologies need to be created that will cover specific types of design and case studies used as examples to classify systems. For example, systems such as pipe networks, electrical circuits and transmission devices all involve the flow of energy (or matter) from component to component. Reliability could be modelled by looking at the number of or connections between the components. The multi-objective method can be used in conceptual design to identify the most critical components (or subsystems) that require reliability improvement.

An option for the designer is to classify systems to permit the use of more than one reliability improvement methodology i.e. guidance for the less experienced designer but versatility for the confident user. This option will allow the user to decide the level of detailed analysis that the system is subjected to, because some systems will require in depth study and others will require less depth.

System classification will be dependent upon the design stage that has been reached. When detail design is undertaken more analytical tools may provide the best solution to reliability assessment. However, in the earlier stages of design qualitative tools will provide the best solution. Because within the scope of this problem only the early design stage is considered this classification will not be covered. But it should be appreciated that it exists.

Methodology will be developed to ask pertinent questions to ascertain which tools and techniques should be utilised on the system to be analysed. It may even suggest that tools that are currently available cannot be used, this will demonstrate the need for further work. It is not anticipated that all system types can be classified but it is hoped that the skeleton of the technique can be constructed.

\subsection{Required data for system}

The type of data required would be dependant upon how the system has been classified. For example, in the case of energy flow classification, information on component type, number of components and the connections between them will be required. Iteration is made back to the specification to provide information regarding firm and loose connections between the components (to allow later changes to be made for reliability improvements). The multi-objective method requires minimum and maximum performance levels of each subsystem (or component at a lower level).

However, for all analysis methods, it is important to remember that at the conceptual design stage the designer has very little detailed information to work from. Therefore the tools developed will need to work with data such as performance boundaries. These performance boundaries could range from load carrying capacity to the reliability requirement of the system as a whole. If complex and accurate data is required for the tools to operate, as is the situation in most cases at present, then the designer will simply not utilise the tools at the conceptual stage. 


\subsection{Modelling methodology}

Dependent upon the number of system classifications available, both old and new reliability methodologies and tools will be made available in the RA Toolbox to model the systems. It is hoped that the more traditional methods such as FMEA and FTA can be integrated with tools specifically developed for this application.

The conceptual design stage must demonstrate feasibility to perform the functional requirements. The objective of the RA Toolbox is to provide an effective excursion tool for reliability feasibility. In order to bridge the gap between the reliability analyst and the multi-disciplinary designer (which is often neglected when tools are created or described), all tools/methodologies should be understandable and be usable without research standard specialist knowledge.

\subsection{Iterative improvements}

Design can be innovative or adaptive. Innovative design would be hard to generate from methodology alone therefore the designer must input initial ideas. Adaptive design could then be employed by methodology that utilises iterative improvements to the original idea.

Carter's work [12] concentrates on the relationship between mechanical load and mechanical strength of the item in question. This is achieved by constructing probability density functions of load, L, and strength, S, shown in Fig. 6a. Failure will only occur in the overlap region, which is related to the safety margin of the item.

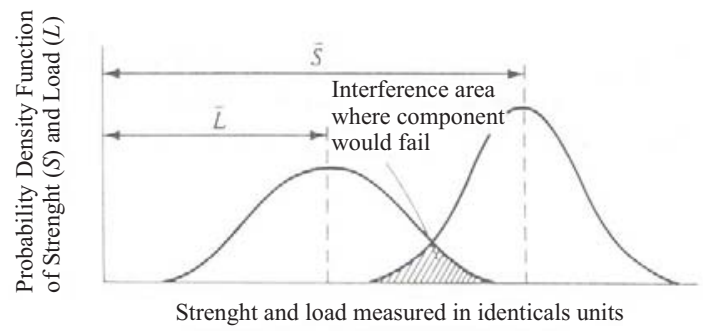

Fig. 6a: Load-strength interference diagram with interference

It is hypothesised by the author that this relationship between load, strength and failure can be extended beyond the mechanical into all functional requirements of a product. Functional requirements could be anything relevant to the product design specification, e.g. weight, speed, size, flow rate, fuel consumption, etc.

By extending Carter's principle, all the functional requirements can be plotted in terms of load and strength, or as it will now be called, requirement and performance. The remainder of this section details the mathematical treatment required to deal with a functional requirement in order to maximise its reliability.

The requirement is to be represented as a probability density function, pdf. However, this raises the question of which distribution to use. It was decided that due to the limited information available at the conceptual design stage that the normal distribution would provide the best approximation as it can be described by just a mean and standard deviation.

In some cases the requirement will be given by a mean, $\mu$, and a standard deviation, $\sigma$. But in most cases limits of perfor- mance will be available from the specification. An upper limit, $Q_{\text {Upper, }}$ above which no improvement in the product will be made. And a lower limit, $Q_{\text {Lower }}$, below which the product will not perform acceptably.

The mean and standard deviation are given by Eqs. (1a) and (1b):

$$
\mu=Q_{\text {Lower }}+k \cdot\left(Q_{\text {Upper }}-Q_{\text {Lower }}\right)
$$

where $0 \leq k \leq 1$, usually $k=0.5$ if no skew is desired

$$
\sigma=\frac{Q_{\text {Upper }}-\bar{Q}}{z}
$$

where $z=1.3$ for $90 \%$ of the distribution must be between the limits. Taken from standard culmulative normal distribution tables [15].

The safety margin, SM, is given by Eq. 2 .

$$
S M=\frac{\bar{P}-\bar{Q}}{\sqrt{\sigma_{P}^{2}+\sigma_{Q}^{2}}}
$$

$\bar{P}=$ mean performance,

$\bar{Q}=$ mean requirement,

$\sigma_{i}=$ i's standard deviation.

Carter [12] postulates that reliability can also be derived from these pdf's. And is given by:

$$
R(t)=\int_{0}^{\infty}\left\{P(s)\left[\int_{0}^{s} Q(s) \mathrm{d} s\right]^{k t}\right\} \mathrm{ds}
$$

where $k=$ constant.

If it is assumed that the reliability is for a given time period, that is time/design life is fixed, then the hazard rate associated with the calculated reliability can be derived from:

$$
R=e^{-\lambda t}
$$

where $t=$ design life.

Within the design specification the requirements are fixed, however, the performance of the product will vary dependant upon what conceptual solutions are formulated by

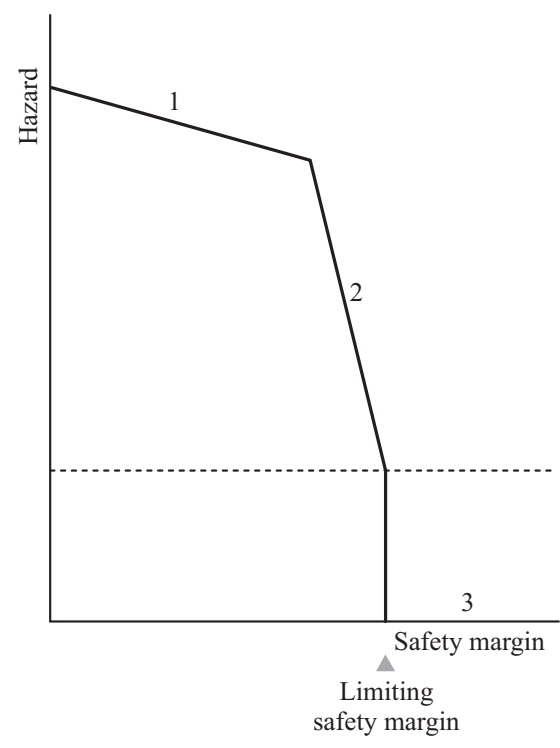

Fig. 6b: Graph of hazard rate verses safety margin 
the designer. If this idea is extended by saying that the requirement pdf is fixed and the performance pdf changes then a graph can be plotted of safety margin verses hazard rate to illustrate their relationship. Fig. 6b shows an approximation of this graph, which is split up into 3 distinct regions [12].

Within the first region the failure rate is too high for practical use. The second region has great instability, showing rapid changes in hazard rate for small increases in safety margin. This is therefore too sensitive and unacceptable.

Region 3 displays intrinsic reliability because the hazard rate has tended to an insignificant value and increasing the safety margin above the limiting value of safety margin has no effect on the hazard rate.

So a limiting safety margin can be found that will make a functional constraint intrinsically reliable. In practice a limiting or allowable hazard rate may need to be defined by the designer before the limiting safety margin can be calculated.

Full product functional reliability can therefore be achieved by using the above methodology to state limiting safety margins, and hence performance requirements, for each major functional design parameter.

Within the design space all functional constraints can be drawn together to form a region of acceptable solutions [16]. Fig. 7 shows the limiting safety margin idea in operation within the design space. Note that not all the limiting safety margins are the same for all the functional constraints. Also that there is not one point which is the perfect solution.

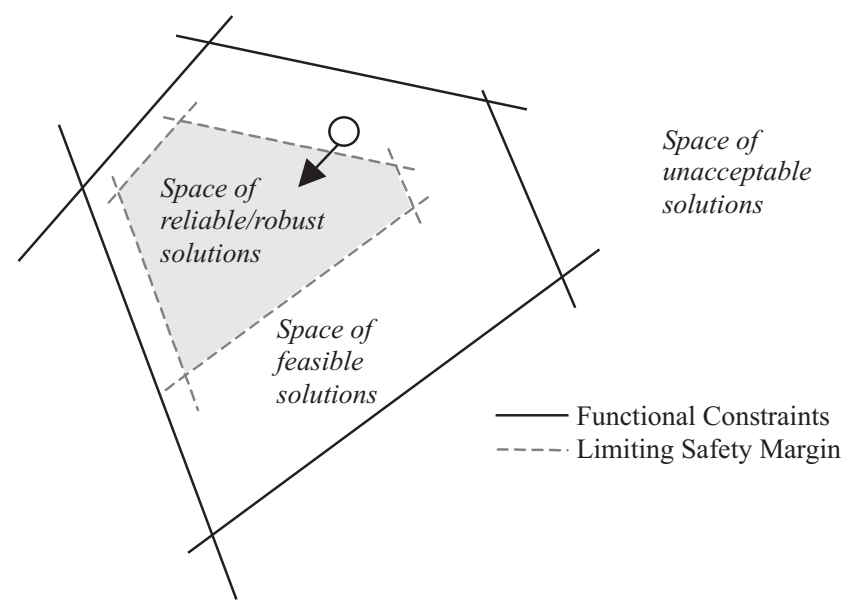

Fig. 7: Iterative algorithm operating in the design space to move towards the robust solution

Provided that the conceptual design falls within the space of reliable/robust solutions it will adequately satisfy any functional reliability requirements. So it can be seen that a number of different solutions can satisfy the design requirements and the idea of the perfect solution, produced by optimisation is fictitious.

The iterative methods will aim to adopt this concept to facilitate concept improvement. It is anticipated that Genetic Algorithms can be made use of to facilitate iterative improvement methods as described by references [17], [18]. These will enable large combinations to be tried, tested, rejected or accepted on the basis of pre-specified Goal Functions.

\subsection{Results representation}

Data or results must be displayed in a user-friendly way. It is of little use to the designer if the tools just indicate the overall reliability. It would be more informative to indicate how the reliability is achieved and what are the most sensitive areas of the design that cause the reliability to increase or reduce. Hence the system could be rethought or experience gained of how to design better for reliability. Again data representation will depend upon the classification of the system. The IT package should have the facility to display results both pictorially (using fault trees or graphs etc.) or in numerical data form. Also design improvement suggestions will be made with regard to reliability.

\section{Example: Solid/fluid separation}

\subsection{Problem background}

Equipment is required to perform a solid/liquid separation function as part of a larger process for a chemical plant. Particulates must be separated from a sulphuric acid solution to produce a pure product. A high reliability is required, as the plant cannot operate without this process functioning continuously. It has been decided that a reliability of 0.8 is required for a 3-month operating period, prior to shutdown for routine maintenance.

Some preliminary design work has been completed but the design required further reliability improvement/assessment prior to starting detail design.

Fig. 8 shows the proposed conceptual solution. It can be seen that the design will perform the essential functions of:

1. Input solid/fluid mixture

2. Remove fluid

3. Remove solid

It can be seen that a centrifuge concept has been used which has the additional function of:

4. Rotate drum

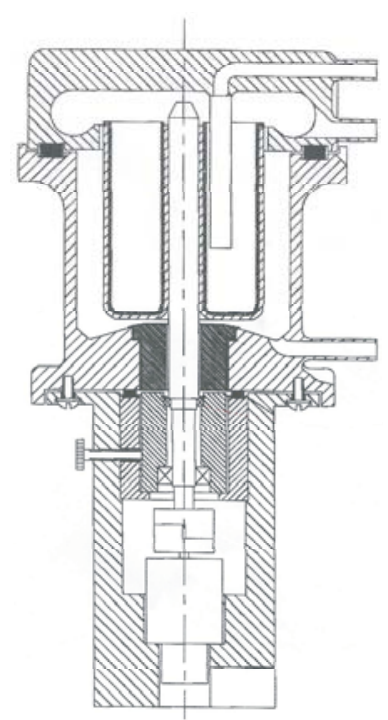

Fig. 8: Sectioned view of a conceptual centrifuge design 


\subsection{Applying the methodology}

Below the methodology is applied sequentially to the problem described in section 4.1. The following is provided as a demonstration, not as a full explanation of the technique.

\section{System classification}

The problem is at the later stages of the conceptual design and would benefit little from reliability allocation or further system reliability analysis. However, if the concept could be improved with respect to its functional performance then reliability of the concept could increase.

Therefore iterative improvement will be attempted.

\section{Required data}

The required data is related to the functional constraints for the design. Requirement (load) and Performance (strength) data will be required for each constraint. These are listed in Table 2. Note that functional constraints have been derived from the 4 main functions listed within the problem background and that limits of performance and requirement have been used.

\section{Results representation}

Safety margin can be calculated for each of the categories shown in Table 2 using equation 1. Plotting varying safety margin against hazard can derive the limiting safety margin. It can then be shown where the design lies within the design space. From this improvements maybe suggested for the centrifuge design. For a more complicated problem algorithms may be appropriate to change the design and note the

effects of all of the parameters. The results of the calculations are shown in Table 2 and illustrated in Fig. 9.

In Fig. 9, the solution is identified by the area described by the initial envelope of performance. It can be seen that this envelope of performance of the proposed solution does lie within the boundaries of feasibility. However, it is not a robust solution. In Fig. 9 the envelope of reliable/robust solutions is much smaller than that of the space of acceptable solutions and the performance envelope of the proposed solution lies outside it. Improvements to the design are therefore required to improve the robustness and thus make the design solution move within the space of reliable/robust solutions.

\section{Iterative improvement}

Fig. 9 clearly shows that the time between solid removal is acceptable within the design limits. Also it can be seen that other functional parameters are unacceptable. For the purposes of this example the solid removal function will be concentrated on. But the principle of application of iterative improvement is the same for all functional parameters.

Possible solid removal solutions must now be investigated to increase the safety margin with respect to this constraint. Care must be taken not to decrease other constraints safety margins below their limiting levels.

Possible solutions investigated are shown below

Run 2 centrifuges in parallel

Increase drum size, larger area to collect solid
- very expensive

- possible but drive mechanism may need to be upsized

Table 2: Main input data and results of the analysis of the centrifuge concept

\begin{tabular}{|c|c|c|c|c|c|}
\hline \multicolumn{2}{|l|}{ Number } & 1 & 2 & 3 & 4 \\
\hline \multicolumn{2}{|l|}{ Function } & Input solid/fluid mixture & Remove fluid & Remove solid & Rotate drum \\
\hline \multicolumn{2}{|l|}{ Measure } & $\begin{array}{c}\text { flow rate } \\
\text { [litre/minute] }\end{array}$ & $\begin{array}{c}\text { flow rate } \\
{[\text { litre/minute] }}\end{array}$ & $\begin{array}{l}\text { solid removal, } \\
\text { [hours] }\end{array}$ & $\begin{array}{c}\text { drum acceleration, } \\
{[\mathrm{g}]}\end{array}$ \\
\hline \multirow[t]{4}{*}{ Requirement } & upper & 4 & 4 & 5 & 1600 \\
\hline & lower & 3 & 3 & 4 & 1000 \\
\hline & mean & 3.5 & 3.5 & 4.5 & 1300 \\
\hline & st. deviation & 0.38 & 0.38 & 0.38 & 230.77 \\
\hline \multirow[t]{4}{*}{ Performance } & upper & 4.5 & 4.5 & 5.5 & 1650 \\
\hline & lower & 3.5 & 3.5 & 4 & 1500 \\
\hline & mean & 4 & 4 & 4.75 & 1575 \\
\hline & st. deviation & 0.38 & 0.38 & 0.58 & 57.69 \\
\hline \multicolumn{2}{|l|}{ Design life } & 3500 & 3500 & 3500 & 3500 \\
\hline \multicolumn{2}{|l|}{ Safety margin } & 0.92 & 0.92 & 0.36 & 1.16 \\
\hline \multicolumn{2}{|l|}{ Reliability } & 0.821 & 0.821 & 0.631 & 0.862 \\
\hline \multicolumn{2}{|l|}{ Hazard rate } & $5.64 \mathrm{E}-05$ & $5.64 \mathrm{E}-05$ & $1.32 \mathrm{E}-04$ & $4.24 \mathrm{E}-05$ \\
\hline \multicolumn{2}{|l|}{ Limiting S.M. } & 5.9 & 5.9 & 5.4 & 6.25 \\
\hline \multicolumn{2}{|c|}{ Ideal performance } & 6.7 & 6.7 & 8.3 & 2775 \\
\hline
\end{tabular}




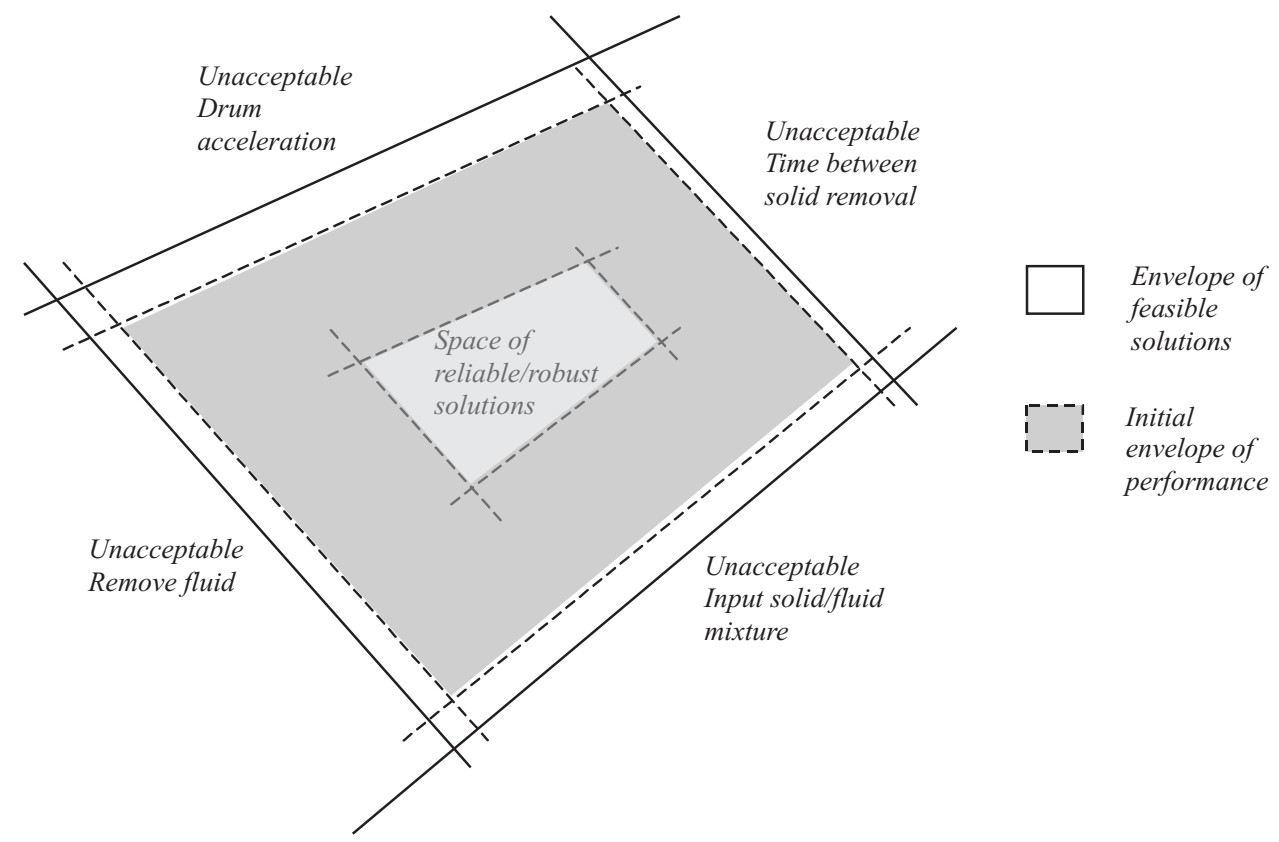

Fig. 9: Functional constraint consideration in the design space to move towards the robust solution

Filter some of solid before it reaches the centrifuge

Calculation showed that the drive mechanism far exceeded the limiting safety margin hence an increase in drum size enables the design to move into the region of robust solutions for this constraint. Table 2 shows the new performance for all the functional parameters and in Fig. 9 the new solution (envelope of performance) would coincides with the limiting safety margin (envelope of requirement). It is pointless to increase the safety margin of any function past the limiting safety margin, as no decrease in hazard rate will be achieved.

\section{Conclusions}

A concept design process is proposed that integrates the development of the design specification (requirements) with concept development in a set of divergent-convergent activities. Excursions are used to investigate important design requirements, e.g. reliability, in order to strengthen the concept in salient respects.

A Reliability Analysis Toolbox is proposed as the method to investigate reliability. The toolbox comprises a systematic process to evaluate the proposed concept and particular reliability analysis methods are applied as required. A review of reliability analysis methods has been undertaken, certain existing methods have been identified as being useful in concept design and other tools show potential for use.

Fig. 10 illustrates the proposed new methodology and shows the impact this will have on the total design process.

It is impossible to produce a methodology that is fully comprehensive and these shortcomings are understood. However, it is envisaged that this methodology could be used as a framework to be added to by others working in this

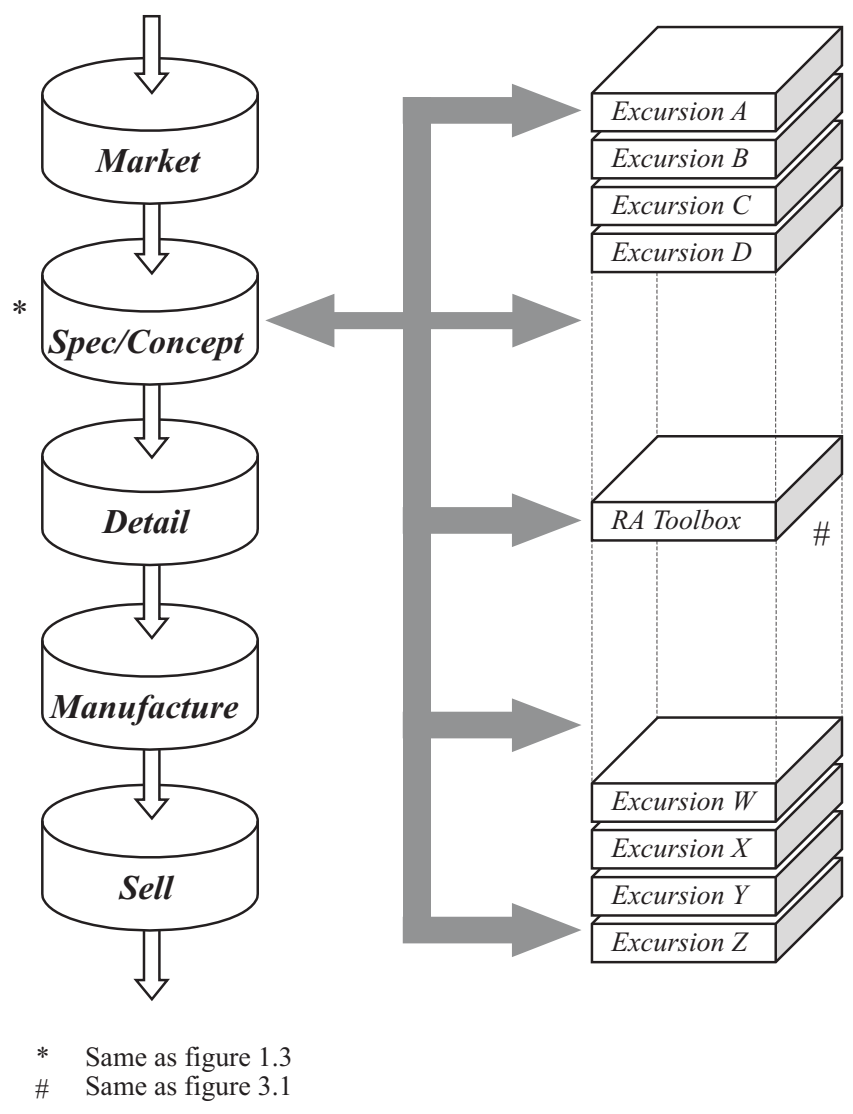

Fig. 10: New methodology in the total design process

field to aid the designer at the conceptual stage with tools, methodologies and IT support. 


\section{References}

[1] Pugh, S.: Total Design - Integrated Methods for Successful Product Engineering. Addison-Wesley Publishing Company, 1991. ISBN 0-201-41639-5.

[2] Thompson, G.: Improving Maintainability and Reliability through Design. London: Professional Engineering Publications, 1999. UK ISBN 1-86058-132-3.

[3] Davidson, J.: The Reliability of Mechanical Systems. Mechanical Engineering Publications Ltd, $2^{\text {nd }}$ Edition, 1994. ISBN 0-85298-881-8.

[4] Andrews, J. D., Moss, T. R.: Reliability and Risk Assessment. Longman Scientific and Technical, 1993. ISBN 0-470-23345-1.

[5] Ridley, L. M., Andrews, J. D.: Reliability of Sequential Systems Using the Cause-Consequence Diagram Method. In: Forteenth International Conference On Advances in Reliability Technology Symposium (ARTS14), Proceedings of the conference held at the University of Manchester $28^{\text {th }}-30^{\text {th }}$ November 2000.

[6] Papadopoulos, Y., McDermid, J., Sasse, R., Heine, G.: Analysis and Synthesis of the Behaviour of Complex Programmable Electronic Systems in Conditions of Failure. Reliability Engineering and System Safety, Elsevier Science, 2001, Vol. 71, No. 3, p. 229-247.

[7] Al-Hakim, L., Kusiak, A., Mathew, J.: A graph-theoretic approach to conceptual design with functional perspectives. Computer aided design, 2000, Vol. 32, No. 14, p. $867-875$.

[8] Sigurdsson, J. H., Walls, L. A., Quigley, J. L.: Using Baysian belief nets in reliability modelling. In: Fourteenth International Conference On Advances in Reliability Technology Symposium (ARTS14). Proceedings of the conference held at the University of Manchester $28^{\text {th }}-30^{\text {th }}$ November 2000.

[9] Thompson, G., Nilsson, C.: A Multi-Objective Approach to Design for Reliability. Accepted for Publication in Institution of Mechanical Engineers Proceedings Part E, Journal of Process Mechanical Engineering, 2001.
[10] Society of Automotive Engineers.: Reliability program standard: Implementation guide JA 1000-1 ... SAE G-11.

[11] Cross, N.: Engineering Design Methods. Chichester: John Wiley, 1994. ISBN 0-471-94228-6.

[12] Carter, ADS.: Mechanical Reliability and Design. Macmillan Press, 1997. ISBN 0-333-69465-1.

[13] Taguchi, G., Elsayed, E. A., Hsiang, T.: Quality Engineering in Production Systems. McGraw-Hill Book Company, 1989. ISBN 0-07-062830-0.

[14] Cheng, Y. L., Wei, H. C., Yuan, J.: On establishment of I/O tables in automation of a fault tree synthesis. Reliability Engineering and System Safety, 1993, Issue 40, p. 311-318.

[15] Pearson, E. S., Hartley, H. O.: Biometrika Tables for Statisticians. $3^{\text {rd }}$ Edition. Cambridge (UK): Cambridge University Press, 1966.

[16] Liu, J. S., Thompson, G., Clarkson, P. J., Parks, G. T.: Design for structural performance by multi-factor optimisation. First ASMO UK/ISSMO Conference on Engineering Design Optimisation, Ilkley (UK), July 1999.

[17] Pattison, R. L., Andrews, J. D.: Genetic Algorithms in Optimal Safety System Design. IMechE Proceedings Part E, Journal of Process Mechanical Engineering, 1999, Vol. E2, p. 111-121.

[18] Goldburg, D. F.: Genetic Algorithms in Search Optimisation and Machine Learning. Addison-Wesley Publishing Company, 1989. ISBN 0-201-15767-5.

Glen Cooper BEng, MSc

e-mail: glen.cooper@student.umist.ac.uk

G.Thompson MSc, PhD, C.Eng., F.I.Mech.E.

e-mail: graham.thompson@umist.ac.uk

phone: +4401612003750 Ext2406, +4401612003846

Mechanical, Aerospace \& Manufacturing Engineering

Department

UMIST, PO Box 88

Manchester, M60 1QD

England, UK 